\title{
R\&D for Neutron Physics
}

\author{
Katsuya Hirota*t \\ Department of Physics, Nagoya University, Furo-cho, Chikusa-ku, Nagoya, 464-8602, Japan \\ E-mail: hirotadphi.phys.nagoya-u.ac.jp
}

Optically controlled slow neutrons are applied to study weak interaction, gravitational interaction and exotic interactions related to new physics beyond the standard model. The efficacy of the neutron beam is increasing by the neutron optics and the application is spreading to fundamental physics and industrial uses. The multilayers neutron mirrors and high counting rate neutron detectors are developing. The Nagoya University Accelerator-driven Neutron Source (NUANS) is also now constructing at main campus of Nagoya University. The aims of this neutron source are 1) Development of BNCT(Boron Neutron Capture Therapy) system, 2) neutron imaging system, 3) neutron device development.

The 3rd International Symposium on "Quest for the Origin of Particles and the Universe" 5-7 January 2017

Nagoya University, Japan

\footnotetext{
* Speaker.

${ }^{\dagger}$ Speaker.
} 


\section{Introduction}

Neutron is chargeless hadron with the lifetime of about 15 minutes. Slow neutrons are available to control their trajectory by using magnetic lenses and multilayer metal mirrors. Optically controlled slow neutrons are applied to study weak interaction, gravitational interaction and exotic interactions related to new physics beyond the standard model. The efficacy of the neutron beam is increasing by the neutron optics and the application is spreading to fundamental physics and industrial uses. However, the use of neutron beams is currently limited to large-scale facilities such as nuclear research reactors and high-intensity accelerators. Applicability of small-scale and mediumscale neutron sources has been discussed for activating the potentials []. A few small-scale neutron sources have been constructed, some others are under construction or under discussion in Japan. These sources are designed relatively specialized for specific applications. The Nagoya University Accelerator-driven Neutron Source (NUANS) is also now constructing at main campus of Nagoya University. The combination of large-scale neutron sources and (small-scale) application-oriented specialized sources is becoming more important. In this paper I explain the current status of our device development and construction of NUANS.

\section{Neutron Device Development}

The Neutron devices such as neutron detectors and mirrors are very important for high accuracy measurement. The high count rate neutron detectors have also important roles for T-violation search measurement by using compound nucleus. Figure $\mathbb{W}$ shows the neutron beam intensity estimation[四], which is calculate from neutron beam measurement at J-PARC/MLF BL04. The yellow line area shows the energy region using for our research mainly. For the T-violation search by using $\mathrm{p}$-wave resonance of lanthanum case, $\mathrm{E}_{n}=0.7$ to $0.8 \mathrm{eV}$ region is most important. This energy neutrons are coming the period of $120 \mu \mathrm{sec}$ when we use J-PARC/MLF BL04. The neutron intensity of this energy region is about $10^{5} \mathrm{n} / \mathrm{eV} / \mathrm{cm}^{2} / \mathrm{pulse}$, which is corresponding to 160 $\mathrm{Mcps} / \mathrm{cm}^{2}$. For the $\mathrm{p}$-wave resonance of xenon case, the incident beam intensity is corresponding to $420 \mathrm{Mcps} / \mathrm{cm}^{2}$. To use for larger neutron beam size, the counting rate requirement is more than $\mathrm{GHz}$ for whole detector area.

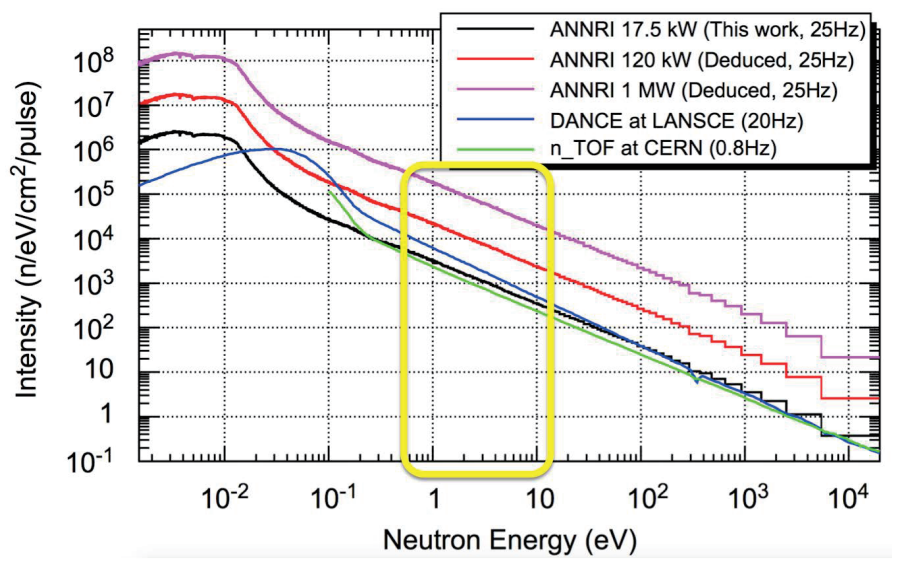

Figure 1: Neutron beam intensity at J-PARC BL04 
Normally used neutron detectors are $\mathrm{ZnS} /{ }^{6} \mathrm{LiF}$, Li glass scintillators and ${ }^{3} \mathrm{He}$ gas detectors, however the time constant of these detectors are order of $1 \mu \mathrm{sec}$ and these are not sufficient for high counting rate detector. The faster decay time of the scintillator are required for T-violation search measurement. The candidates material of the detector develop is boron-loaded liquid scintillator, like BC523A. This scintillator has decay time of $3.7 \mathrm{nsec}$. The LBO:Ce scintillator is also good candidate. This scintillator has very short time decay constant of order of $1 \mathrm{nsec}$, but the light emission is also very small, less than $1 \%$ of anthracene light emission. It is difficult to operate so this development is future plan.

The multilayer neutron mirrors are also developed by using DC magnetron sputtering system. The $\mathrm{Ni}$ and $\mathrm{Cr}$ multilayer mirror is the best combination for unpolarized neutron beam mirror because of the difference of the optical potential value. The DC magnetron sputtering system has an advantage that the sputtering rate is high and it will be easy to make large neutron mirrors. However the $\mathrm{Cr}$ is magnetic material and it is difficult to operate by this system. To overcome this problem NiCr alloy is used for this system.

\section{Construction of Nagoya University Accelerator-driven Neutron Source, NUANS}

The Nagoya University Accelerator-driven Neutron Source, NUANS is now constructing in the main campus of NagoyaUniversity. The aims of this neutron source are 1) Development of BNCT(Boron Neutron Capture Therapy) system, 2) neutron imaging system, 3) neutron device development. The figure $\square$ shows the schematic view of NUANS. Our constructing plan has two beam lines in NUANS. The first beam line is planning to use for BNCT system development, and the second beamline is for neutron imaging and neutron device development. In this paper the explanation is focusing to the second beamline.

The dynamitron accelerator, which is electrostatic machine, and ECR ion source are used for this neutron source. The maximum proton energy is $\mathrm{E}_{p}=2.8 \mathrm{MeV}$, maximum current is $\mathrm{I}_{p}=15 \mathrm{~mA}$ and the corresponding power is $42 \mathrm{~kW}$.

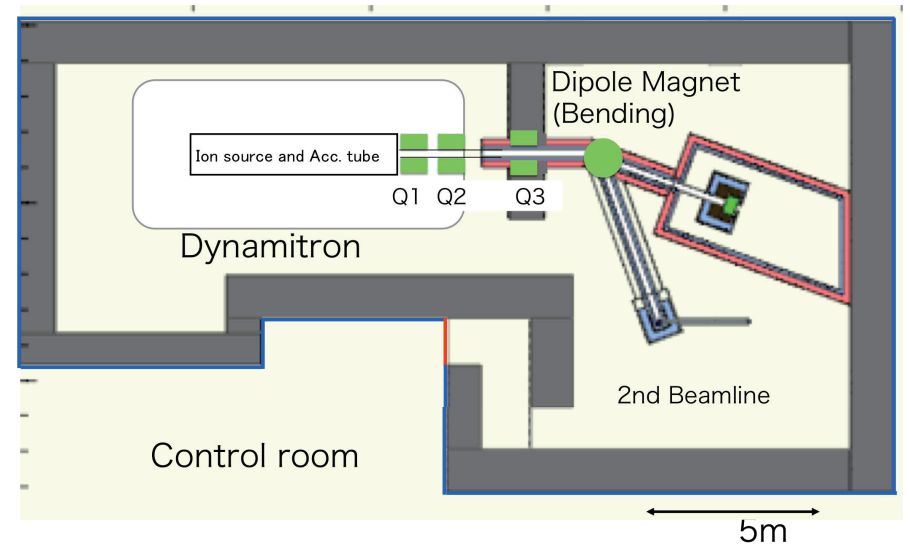

Figure 2: Schematic view of NUANS

The neutron emission target is one of the important devices on neutron source. The neutron emission yield was studied for several target materials and incident beams[[B]. The $\mathrm{Li}$ and $\mathrm{Be}$ are 
the candidate of the neutron emission target at low energy proton beam. The neutron emission yield from ${ }^{7} \mathrm{Li}$ is about one order larger than ${ }^{9} \mathrm{Be}$ at $\mathrm{E}_{p}=2.8 \mathrm{MeV}$, however Li metal is chemically unstable and it is difficult to use for neutron target. We are planning to develop this Li target using 1st beamline. The $\mathrm{Be}$ is chemically more stable than $\mathrm{Li}$ and it is suitable for using neutron emission target regardless of lower neutron emission yield. In this reason we decide to use this Be target for 2nd beamline. As Be target has also difficult technical point, many of facilities are developing these neutron emission target and some of the targets were broken[వ]. RIKEN group is success to develop this target[团]. We used this RIKEN technique and applied to NUANS accelerator conditions.

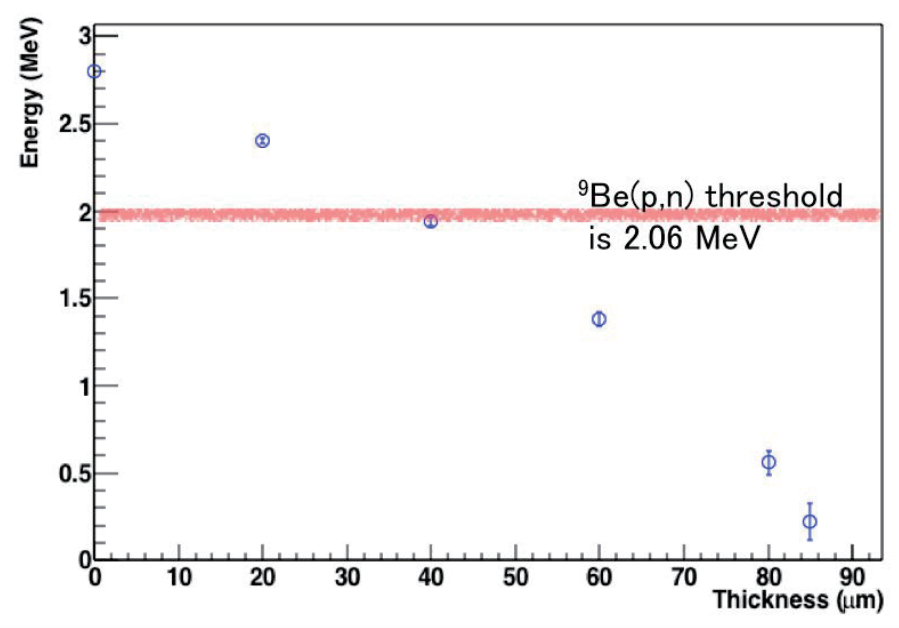

Figure 3: proton energy in Be foil

Figure [3 shows the incident proton energy in Be foil calculated by SRIM[[]]. The incident proton beam energy of $2.8 \mathrm{MeV}$ is decreasing with foil thickness and the energy is below $\mathrm{Be}(\mathrm{p}, \mathrm{n})$ threshold energy at $40 \mu \mathrm{m}$ thickness. The $\mathrm{Be}(\mathrm{p}, \mathrm{n})$ reaction is no longer happen after this foil thickness and proton is stopped around $85 \mu \mathrm{m}$. If we use the Be foil more than $85 \mu \mathrm{m}$ thickness, the incident proton beam is stopped in the Be foil and change to hydrogen gas, because the hydrogen diffusion coefficient in Be is very low. After irradiate the proton beam the hydrogen gas make blister in Be foil and neutron target is broken. To prevent from the broken, we choose the target thickness less than $85 \mu \mathrm{m}$. At the result the suitable Be target thickness is $40 \mu \mathrm{m}$ to $85 \mu \mathrm{m}$ in this NUANS condition. This thickness value is of course depend on the incident proton energy.

Figure [3 shows the photograph of Be target for NUANS 2nd beamline. The black circle material is the Be foil of $40 \mu \mathrm{m}$, and around metal color one is vanadium of $5 \mathrm{~mm}$ thickness.

The shielding design of proton and neutron beamlines are calculated by using PHITS code. The result of the 2 nd beamline design is shown in figure $\mathbf{b}$. The proton beam is coming from left side to center in this figure. There is a Be neutron emission target in the center position. The neutron exit angle is about 70 degree. Two kinds of shielding material, boron induced polyethylene and lead blocks are used as three layers shielding structure. The size and weight reduction are achieved by placing lead block as a middle layer. As a result, the shield around the neutron emission target can be set to $100 \mathrm{~cm}$ on a side, and the measurement port can be installed at $50 \mathrm{~cm}$ from the neutron target. 


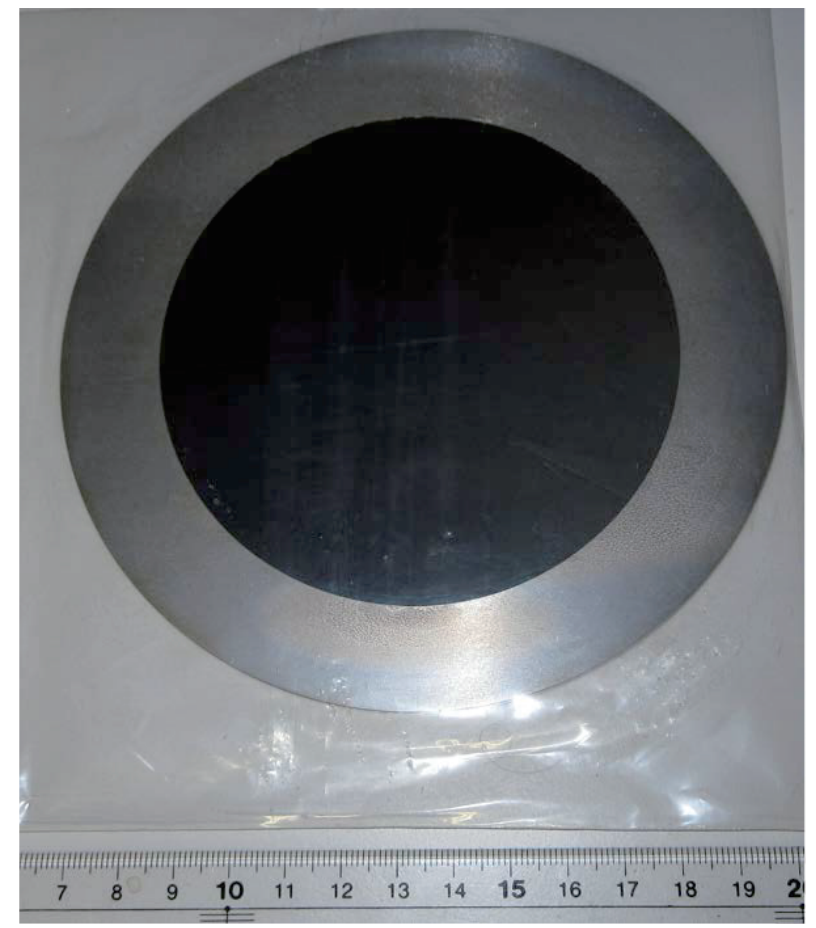

Figure 4: Neutron emission target

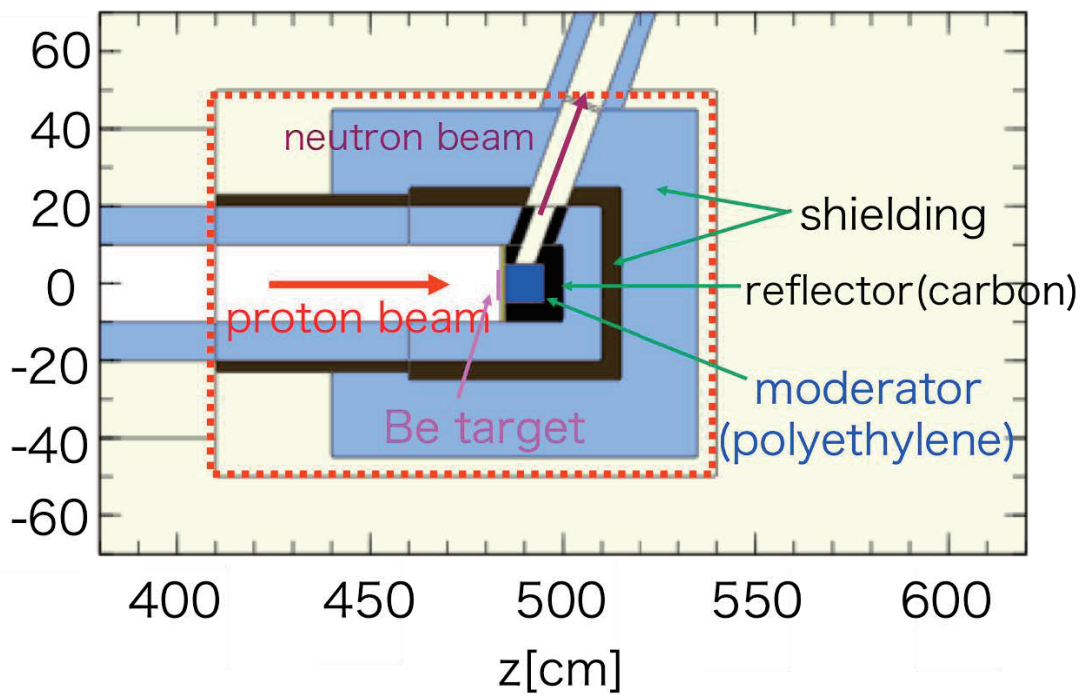

Figure 5: Neutron emission target

The neutron intensity is also calculated using PHITS under the condition that an imaging detector (scintillator + CCD detector) installed just outside the shield. Figure 6 shows the result for the neutron beam intensity distribution. The maximum intensity is the central area and minimum is the peripheral, and the difference is about $25 \%$, which value is acceptable for imaging measurement. The maximum intensity is about $10^{6} \mathrm{n} / \mathrm{cm}^{2} / \mathrm{sec}$. This neutron intensity is suitable for our 


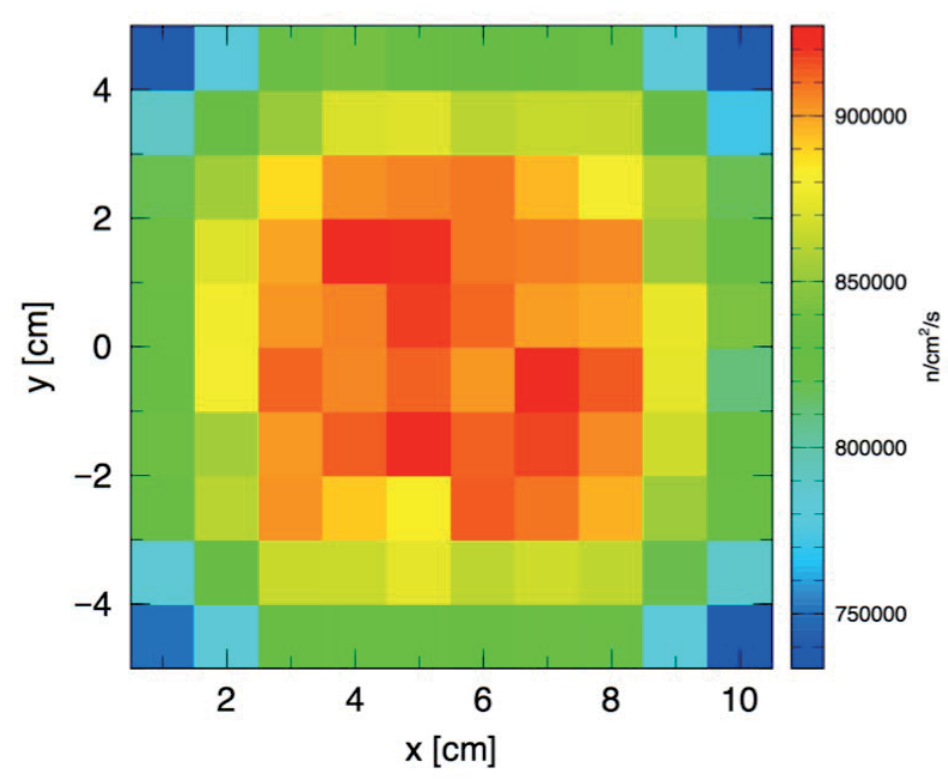

Figure 6: Neutron beam distribution

neutron imaging and detector development.

\section{References}

[1] K. Kino, M. Furusaka, F. Hiraga, T. Kamiyama, Y. Kiyanagi, K. Furutaka, S. Goko, H. Harada, M. Harada, T. Kai, A. Kimura, T. Kin, F. Kitatani, M. Koizumi, F. Maekawa, S. Meigo, S. Nakamura, M. Ooi, M. Ohta, M. Oshima, Y. Toh, M. Igashira, T. Katabuchi, M. Mizumoto NIM A626-627(2011)58

[2] I.S. Anderson, C. Andreani, J. M. Carpentaer, G. Festa, G. Gorini, C. K. Loong and R. Senesi. Phys. Rep. 654 (2016) 1-58

[3] M.R. Hawkesworth, Neutron Radiography: Equipment and Methods, Atomic Energy Review 15, No. 2, 169-220, 1977.

[4] Yutaka Yamagata, Katsuya Hirota, Jungmyoung Ju, Sheng Wang, Shin-ya Morita, Jun-ichi Kato, Yoshie Otake, Atsushi Taketani, Yoshichika Seki, Masako Yamada, Hideo Ota, Unico Bautista, Oinngan Jia. J Rad. Nucl. Chem. (2015) 305: 787-794

[5] http://www.srim.org

[6] T. Sato, K. Niita, N. Matsuda, S. Hashimoto, Y. Iwamoto, S. Noda, T. Ogawa, H. Iwase, H. Nakashima, T. Fukahori, K. Okumura, T. Kai, S. Chiba, T. Furuta and L. Sihver, Particle and Heavy Ion Transport Code System PHITS, Version 2.52, J. Nucl. Sci. Technol. 50:9, 913-923 (2013) 